\title{
Multiple Lymphomatous Polyposis of the Gastrointestinal Tract
}

\author{
- A Report of Two Cases, with Immunohistochemical Studies - \\ Ju Hie Lee M.D., So Yeon Yu M.D., Mun Ho Yang M.D., Lin Chang M.D.*, \\ Young Tae Ko M.D.** \\ Departments of Pathology, Medicine*, and Radiology**, Kyung Hee University, School of Medicine, \\ Seoul, Korea

\begin{abstract}
We describe two cases of multiple lymphomatous polyposis in the gastrointestinal tract from the esophagus to the rectum. Clinical findings, histopathologic and immunohistochemical findings in paraffin embedded tissue are discussed. It is important to recognize this rare form of gastrointestinal lymphoma because of the prognostic and therapeutic implications.
\end{abstract}

Key Words: Multiple lymphomatous polyposis, Centrocytic lymphoma, Intermediate lymphocytic lymphoma, Gastrointestinal lymphoma

\section{INTRODUCTION}

The gastrointestinal (Gl) tract is the most common site of extranodal malignant lymphomas and accounts for about $13.7 \%-26.7 \%$ of the lymphomas in Korea (Lee et al., 1973; Lee and Lee, 1982). Primary Gl lymphomas most commonly present as a single lesion. In $4.3 \%$ of cases, lymphoma occured as two to six separate nodules at one anatomic location and in $2.6 \%$ were described as multiple lymphomatous polyposis involving either the colon or small intestine (Lewin et al., 1978). The term "multiple lymphomatous polyposis" of the gastrointestinal tract was introduced by Cornes in 1961 to describe malignant lymphoma that presented as multiple polyposis affecting long segments of the Gl tract. Since that time, investigation of the Gl tract by imaging; endoscopy and biopsy has developed considerably. These advances are reflected in a number of recent reports of multiple lymphomatous polyposis (Davis et al., 1989; Nakamine et al., 1989; Ohri et al., 1989; Jalleh et al., 1990).

Recently we observed two patients in whom the gross appearance and the pattern of proliferation resembled multiple lymphomatous polyposis, involving the entire

Address for correspondence: Ju-Hie Lee, Department of Pathology, Kyung Hee Univ. Medical Center \#1 Hoegi-dong Dongdaemun-ku Seoul 130-702 Korea Tel: (02) 966-5191 Ext 2558. This paper was presented at the 43rd meeting of the Korean society of pathologists. gastrointestinal tract. To our knowledge this is the first report of multiple lymphomatous polyposis in Korea that has been studied by immunohistochemical method.

\section{CASE PRESENTATION}

The clinical findings are summarized in Table 1. Patient 1, a 64 year old man, presented with bloody diarrhea and weight loss. Colon study revealed numerous filling defects involving the entire colon and rectum (Fig. 1). Three months after the initial symptoms appeared, an exploratory laparatomy was done under the clinical impression of familial polyposis. Frozen section diagnosis was malignant lymphoma and an open biopsy of the ileum, transverse colon, appendix and regional lymph nodes was done. The involvement of the stomach, duodenum and esophagus was found by an upper gastrointestinal study and endoscopic examination (Fig. 3) followed by biopsy. He was treated with 6 cycles of CHOP-Bleomycin. He had only partial remission. Two years later a bone marrow biopsy showed focal involvement by lymphoma. The therapeutic regimen was changed to PRO-MACE. He had a relapse of cervical lymph node involvement 4 years after diagnosis. He is still alive and undergoing therapy.

Patient 2, a 56 year old man, presented with postprandial epigastric pain. Numerous polypoid or sessile lesions were found in the alimentary tract from the mid esophagus to the rectum by endoscopic examination 
Table 1. Clinical Findings of Multiple Lymphomatous Polyposis

\begin{tabular}{llc}
\hline & Case 1 & Case 2 \\
\hline Clinical Presentation & Bloody diarrhea & Epigastric pain \\
Histology & Centrocytic (?) & Centrocytic (?) \\
Polyposis & Colon, ileum, appendix & Stomach, duodenum \\
$\quad$ at diagnosis & rectum & esophagus, colon \\
Polyposis later & Stomach, esophagus & - \\
Abdominal LN & + & - \\
Peripheral LN & + & - \\
Spleen & Splenomegaly & - \\
Bone marrow & + & - \\
Treatment & CVP, CHOP-Bleomycin & CVP \\
Response & PRO-MACE & Partial response \\
\hline
\end{tabular}

CVP: cytoxan, vincristine, prednisone

CHOP: cyclophosphamide, vincristine, adriamycin, prednisone

PRO-MACE: prednisone, adriamycin, cytoxan, methotrexate, VP-16

LN: Lymph node

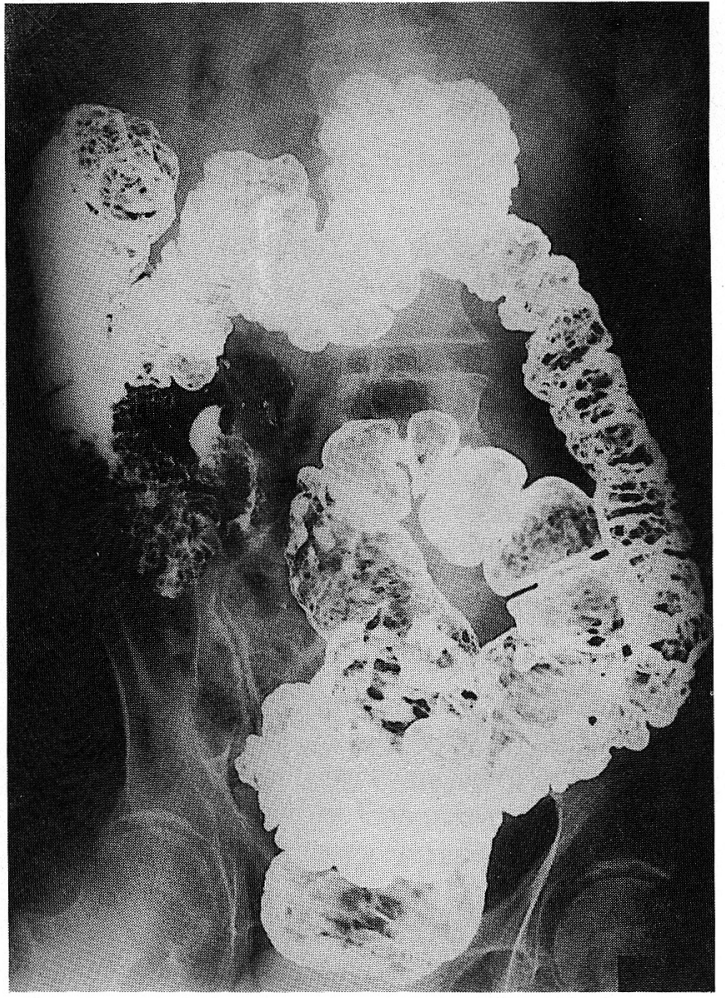

Fig. 1. Colon study reveals multiple nodular defects involving the entire colon and the rectum in patient 1.

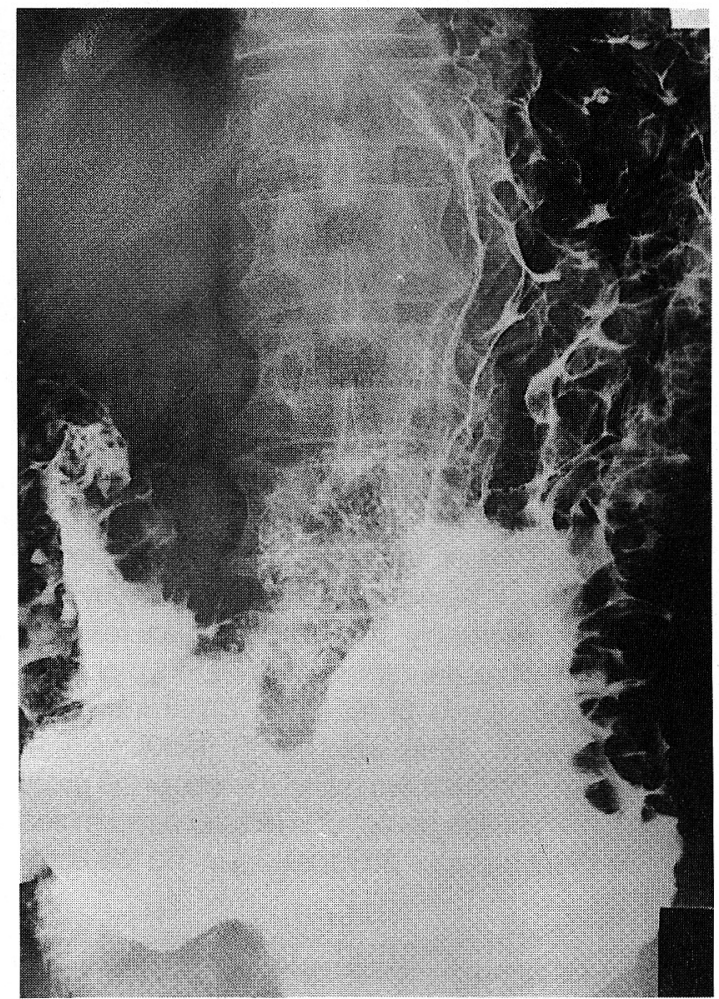

Fig. 2. Upper GI study reveals marked mucosal thickening with nodular defects involving the stomach and the duodenum in patient 2. 


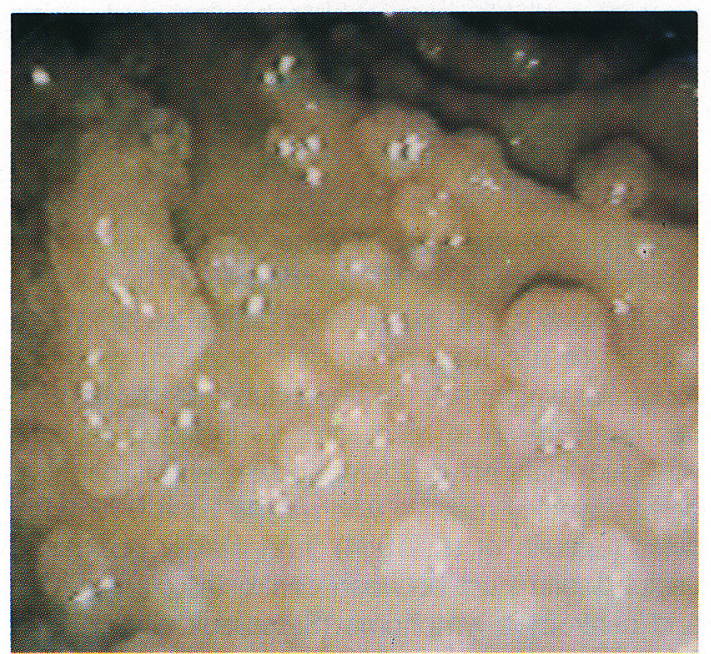

Fig. 3. Endoscopic findings of the duodenum show multiple polypoid elevation of the mucosa in patient 1.

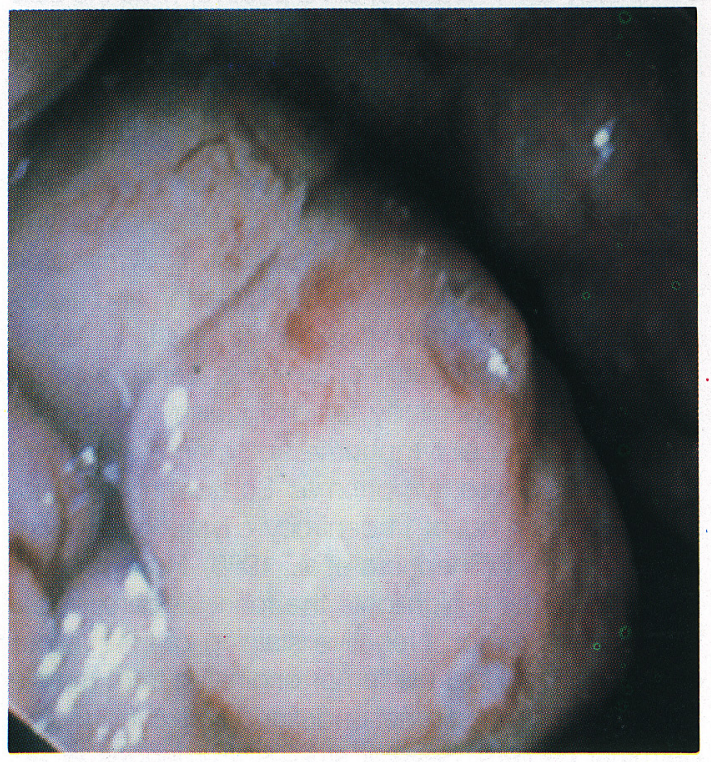

Fig. 4. Endoscopic findings of the stomach show polypoid mass of the mucosa with superficial ulcer in patient 2.

(Fig. 4) and multiple endoscopic biopsy. But the number of lesions was decreased in the colon. An upper gastrointestinal study revealed massive mucosal thickening with several nodular defects in the stomach and esophagus. (Fig. 2) He was treated with CVP. He is still alive and undergoing therapy.

\section{HISTOLOGY AND IMMUNOHISTOCHEMISTRY}

Each polypoid lesion of the gastrointestinal mucosa was composed of small lymphoid cells with a vaguely nodular or diffuse pattern. The lesion was usually confined to the mucosa and submucosa (Fig. 5). Cytologic detail of the lymphoid cells revealed a small round nucleus with an irregular nuclear membrane and scanty or pale cytoplasm (Fig. 6). There were a few mitotic figures. A regional mesenteric lymph node was also involved by the lymphoma with vaguely nodular pattern. Bone marrow examination revealed focal involvement by lymphoma. The cervical lymph node was totally replaced by a lymphoma with a diffuse pattern. In patient 2, the first biopsy shows atypical lymphoid proliferation and a suspicious inactive germinal center, rendering impossible a definite diagnosis of malignant low grade lymphoma. However, the fitth and last biopsy specimen had a diffuse pattern and intermediately differentiated lymphocytic cytologic characteristics. Both were classifiable as poorly differentiated

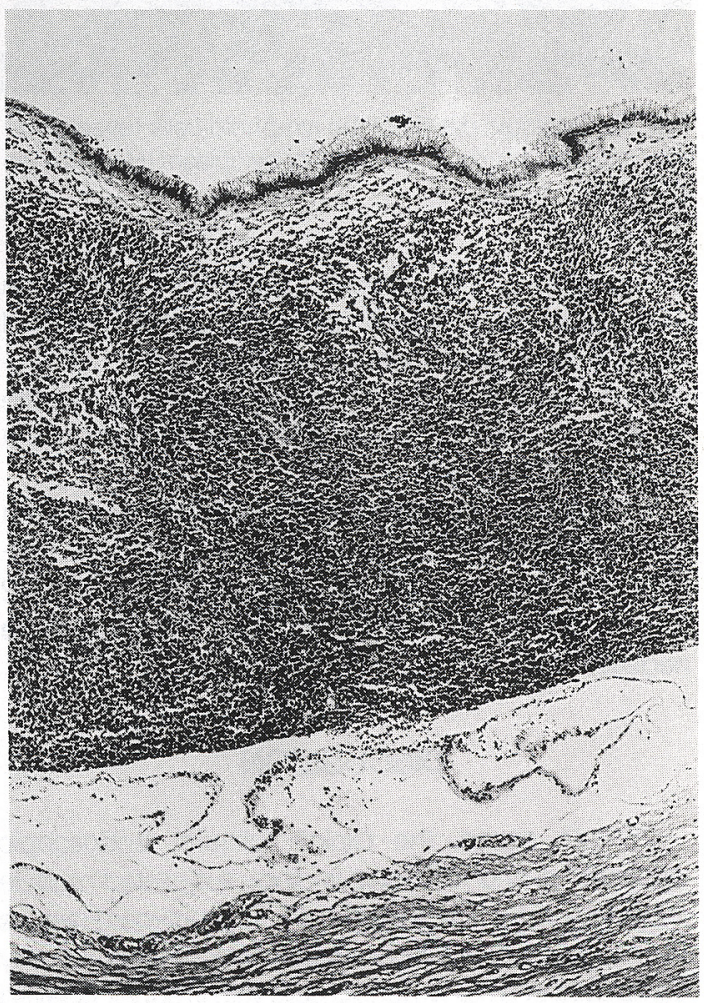

Fig. 5. Lymphoma involves the deep mucosa and submucosa of the appendix with sparing the surface epithelium (Hematoxylin \& Eosin, $\times 40$ ). 


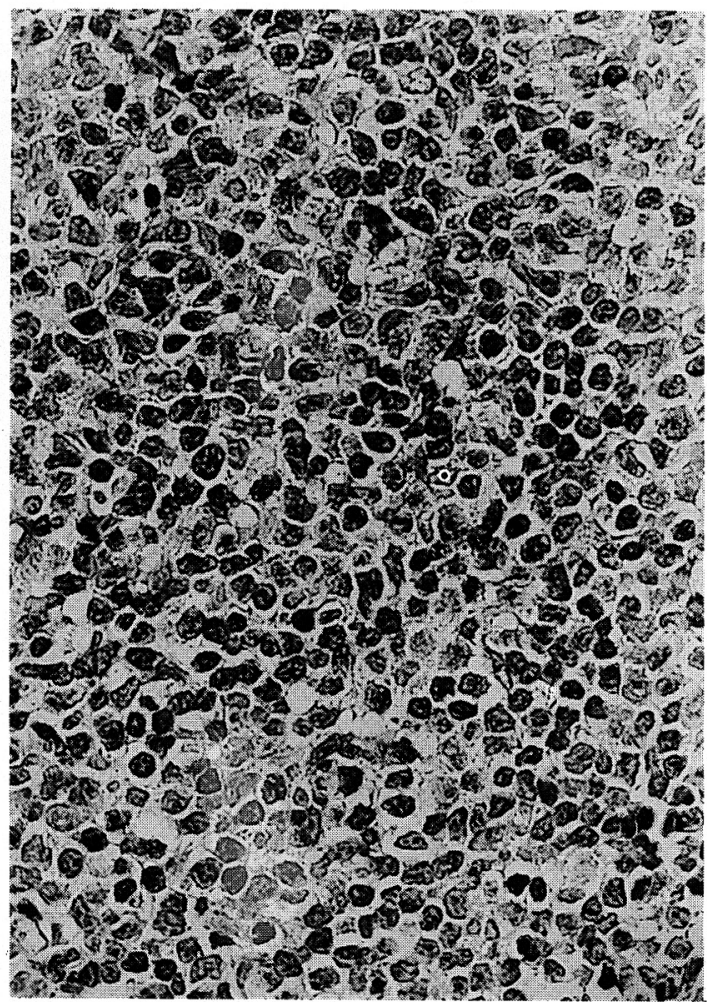

Fig. 6. Cytologic details of the lymphoid cells reveal small round to cleaved nuclei and scanty cytoplasm (Hematoxylin \& Eosin, $\times 400$ ).

lymphocytic or intermediate lymphocytic lymphoma (Rappaport classification), centrocytic lymphoma (Kiel classification), small cleaved follicular center cell lymphoma (Lukes \& Collins classification) and intermediate lymphocytic lymphoma or small cleaved cell lymphoma (Working Formation).

Immunohistochemistry using formalin fixed paraffin tissue confirmed lymphoma of $B$ cell origin. The lymphocytes were positive for L26 (CD20) and LN2 (CD74) UCHL1 (CD45RD) and LN1 (CD $\left.{ }_{w} 73\right)$ were negative in both (Fig. 7).

\section{DISCUSSION}

In both cases, the clinical picture, endoscopic appearance and radiologic findings suggested multiple lymphomatous polyposis (MLP). The polypoid lesions of the gastrointestinal mucosa were microscopically composed of small lymphoid cells with irregular cleaved nuclear membranes. A diffuse and vaguely nodular pattern surrounding an inactive atrophic germinal center is present. Because there was no fresh or frozen material available, immunọistochemical study using monoclonal antibodies as described previously (Isaacson et al., 1984; Fernandes et al., 1985; Triozzi et al., 1986; Zuckerman et al., 1986) could not be done. However, paraffin section immunohistochemistry showed these cells to be positive for $B$ cell related antigens, L26 and LN2. Another follicular center cell related $B$ cell antigen, $L N 1$ was negative in both cases. The antibody to LN1 is a monoclonal mouse IgM which recognizes a surface membrane sialoantigen whose molecular weight has not been clearly identified. LN1 has been found to react strongly in tissue sections with germinal center $B$ lymphocytes and is negative or very weakly reactive with mantle zone B lymphocytes (Epstein et al., 1984). LN1 initially seemed a useful discriminant between centroblastic/centrocytic and centrocytic lymphoma (Samoszuk et al., 1986; Norton and Isaacson 1987). Samoszuk et al (1986) reported that the mantle zone and interfollicular cells in all 6 cases of mantle zone lymphoma were almost completely $\mathrm{LN}-1$ negative, $\mathrm{LN}-2$ positive, while the cells in the residual follicles were uniformly $\mathrm{LN}-1$ positive, LN-2 positive. In the study of sixty three well established B-cell lymphomas, $\mathrm{LN}-1$ was positive in all cases of follicular lymphoma (10/10), diffuse mixed small and large cell lymphoma (2/2), large cell noncleaved lymphoma (8/8) and 4 out of 5 small noncleaved cell lymphoma. However, 5 cases of centrocytic lymphoma were uniformly negative with $\mathrm{LN}-1$ (Norton and Isaacson 1987). The immunostaining with the LN-1, LN-2 and immunoglobulin light chains was therefore considered to have specificity for distinguishing between follicular center cell lymphoma and mantle zone lymphoma/centrocytic lymphoma. Subsequent reports indicated that 4 out of 11 centrocytic lymphomas were positive for LN1 (Shepherd et al., 1988). However LN1 is still useful as it highlights the follicular center cells and derived tumor. Negative staining of LN1 in our cases suggests that the tumor originated from mantle zone cells or from distinct undefined cells surrounding germinal centers. Several studies (Fernandes et al., 1985; Triozzi et al., 1986; Zuckerman et al., 1986) have shown that the neoplastic cells in most MLP cases originate from B cells with special histologic, and immunohistochemical findings similar to those of intermediate lymphocytic lymphoma (ILL) and mantle zone lymphoma (MZL), a follicular variant of intermediate lymphocytic lymphoma. Isaacson et al. (1984) suggested that MLP is a gastrointestinal presentation of ILL/MZL. MLP is clinically distinct from other forms of gastrointestinal malignant lymphoma and results from gastrointesinal involvement by a B cell centrocytic 

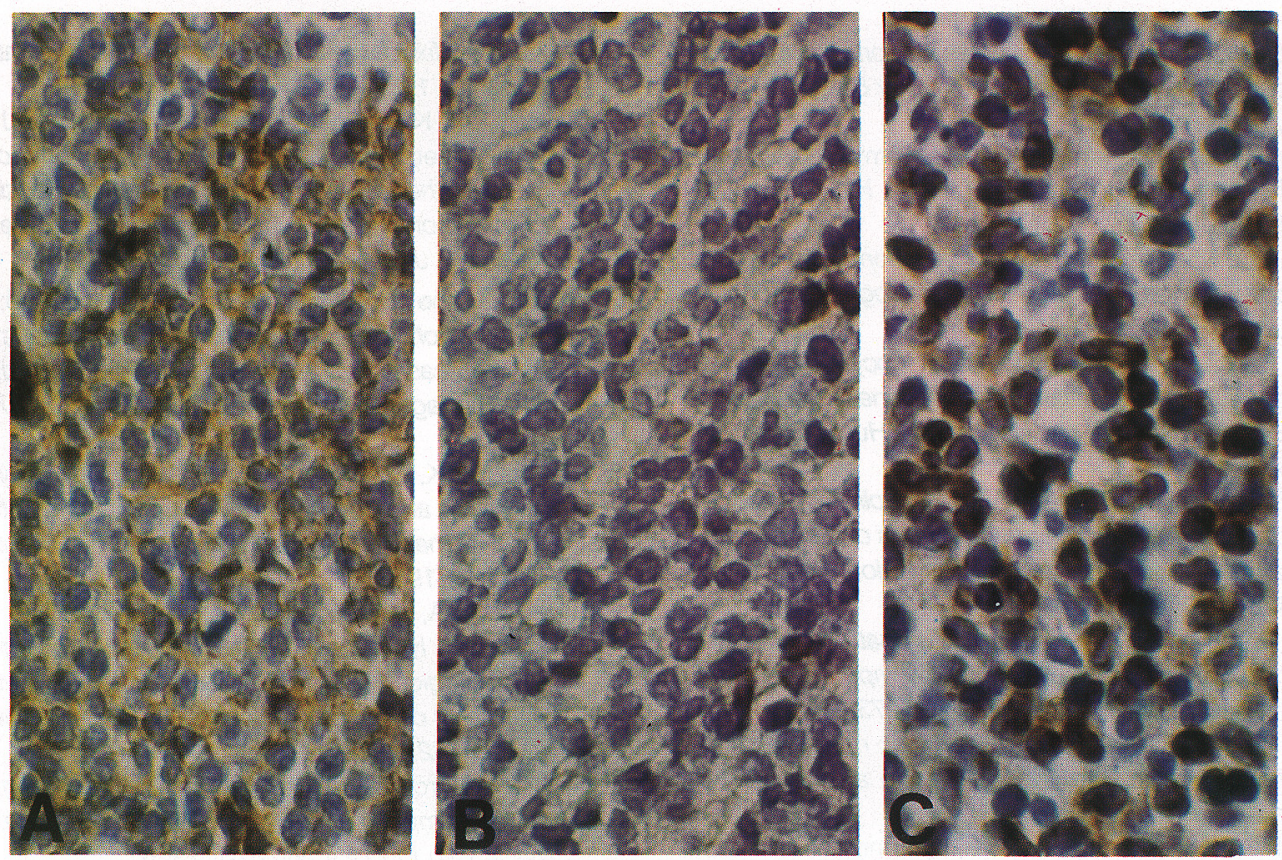

Fig. 7. When stained by avidin-biotin-peroxidase complex method, the tumor cells are positive for L26 (a) and LN2 (b). LN1 is negative (c) ( $\times 200)$.

lymphoma (O'Briain et al., 1989). Some of the cases classified as intermediate lymphocytic or mantle zone lymphoma correspond most closely to the centrocytic lymphoma in the Kiel classification (Jaffe et al., 1987). Whatever the terminology used, all these entities describe a broadly similar disease and are composed of small round to cleaved lymphocytes (centrocytes) in a diffuse pattern or surrounding germinal centers. Immunological studies have revealed B cell lymphoma of IgM or MD type. Lambda light chain excess and coexpression or variable expression of CD5 and CD10 are frequent. It is important to recognize this entity, MLP, since it is usually widespread and clinically aggressive, with a median survival of only 3 years, in contrast to the prolonged survival of most patients with primary gastric lymphoma of mucosa associated lymphoid tissue (Isaacson et al., 1987). Although lymphoma of mucosa associated lymphoid tissue is of B cell type and has been described as centrocyte like, it differs considerably from MLP. It is usually unifocal, frequently shows a plasma cell component and a characteristic lymphoepithelial lesion and is usually CD5 negative. None of these findings were found in our cases. Shepherd et al. (1988) recently reported that surgery was the primary treatment for localized lymphoma of the gastrointestinal tract with radiotherapy and/or chemotherapy for advanced cases. For cases of MLP, chemotherapy is the treatment of choice. Unlike other gastrointestinal lymphomas, MLP do not remain localized to the gut wall and have evidence of distant nodal involvement as in patient 1. Another problem lies in making a diagnosis of low grade lymphoma of the Gl tract with small endoscopic biopsy, as in patient 2. It is often very difficult to distinguish from benign inflammatory lymphoid infiltrates because of the absence of marked cytologic atypia (Zukerberg et al., 1990). Immunohistologic analysis can be useful in distinguishing benign from malignant infiltrates on frozen or paraffin sections. Gene rearrangement can be necessary to establish the nature of the lesion.

\section{REFERENCE}

Cornes JS: Multiple lymphomatous polyposis of the Gl tract. Cancer 14:247-257, 1961

Davis M, Maxwell G, Gogel H, Chen YM, Ott DJ: Lymphomatous polyposis of the colon. Gastrointest Radiol 14:70-72, 1989.

Epstein Al, Marder RJ, Winter JN, Fox RJ: Two new monoclonal $A b(L N 1, L N 2)$ reactive in $B 5$ formalin fixed, paraffin embedded tissue with follicular center and mantle zone human B lymphocytes and derived tumors. $\mathrm{J} / \mathrm{m}$ munol 133:1028-1036, 1984.

Fernandes BJ, Anato D, Goldfinger M: Diffuse lymphoma- 
tous polyposis of the GI tract: A case report with immunohistochemical studies. Gastroenterology 88:1267-1270, 1985.

Isaacson PG, MacLennan KA, Subbuswamy SG: Multiple lymphomatous polyposis of the GI tract. Histopathology 8:641-656, 1984.

Isaacson PG, Spencer J: Malignant lymphoma of mucosaassociated lymphoid tissue. Histopathology 11:445-461, 1987.

Jaffe ES, Bookman MA, Longo DL: Lymphocytic lymphoma of intermediate differentiation-mantle zone lymphoma: a distinct subtype of B cell lymphoma. Hum Pathol 18: 877-880, 1987.

Jalleh RP, Semeraro D, Vellacott KD: Cecocolic intussusception in multiple lymphomatous polyposis of the gastrointestinal tract. Report of a case. Dis Colon Rectum 33: 424-426, 1990.

Lee KK, Lee YB, Kim DS: Clinical, histopathological and histochemical studies on malignant lymphoma among Koreans. Korean J Pathol 7:13-28, 1973.

Lee CS, Lee TS: A pathologic study of non-Hodgkin's lymphoma-around the Chung Cheong area-Korean $J$ Pathol 16:238-242, 1982.

Lewin KJ. Ranchod M. Dorfman RF, Lymphomas of the Gl tract: A study of 117 cases presenting with Gl disease. Cancer 42:693-707, 1978.

Nakamine H, Sakamoto H, Tanaka T, Nakashima N, Takenaka T, Maeda J: UCHL1-positive extranodal lymphoma resembling multiple lymphomatous polyposis of the gastrointestinal tract. Cancer 64:1500-1503, 1989.

Norton AJ, Isaacson PG: Detailed phenotypic analysis of $B$ cell lymphoma using a panel of antibodies reactive in routinely fixed wax-embedded tissue. Am J Pathol 128: 225-240, 1987.

O'Briain DS, Kennedy MJ, Daly PA, O'Brien AAJ, Tanner WA, Rogers $P$ Lawlor E: Multiple lymphomatous polyposis of the Gl tract. A clinicopathologically distinctive form of non-Hodgkin's lymphoma of B-cell centrocytic type. Am J Surg Pathol 13:691-699, 1989.

Ohri SK, Keane PF, Sackier JM, Hutton K, Wood CB: Primary rectal lymphoma and malignant lymphomatous polyposis. Two cases illustrating current methods in diagnosis and management. Dis Colon Rectum 32:1071-1074, 1989.

Samoszuk MK; Epstein AL, Said J. Lukes RJ, Nathwani BN: Sensitivity and specificity of immunostaining in the diagnosis of mantle zone lymphoma. Am J Clin Pathol 85: 557-563, 1986.

Shepherd NA, Hall PA, Coates PJ, Levison DA: Primary malignant lymphoma of the colon and rectum. A histopathological and immunohistochemical analysis of 45 cases with clincopathological correlation. Histopathology 12:235-252, 1988.

Triozzi PL, Borowitz MJ, Gockerman JP: Gl involvement and multiple lymphomatous polyposis in mantle zone lymphoma. J Clin Oncol 4:866-873, 1986.

Zuckerman MJ, Pittman DL, Boman D, Farley PC: Multiple lymphomatous polyposis of the GI tract with immunologic marker studies. J Clin Gastroenterol 8:295-300, 1986.

Zukerberg LR, Ferry JA, Southern JF, Harris NL: Lymphoid infiltrates of the stomach: Evaluation of histologic criteria for the diagnosis of low-grade gastric lymphoma on endoscopic biopsy specimens. Am J Surg Pathol 14: 1087-1099, 1990. 